\title{
Cylindrical and Spherical Membranes of Anodic Aluminum Oxide with Highly Ordered Conical Nanohole Arrays
}

\author{
Yantao Pang1,2*, Rohith Chandrasekar ${ }^{2}$ \\ ${ }^{1}$ School of Science, Shandong Jianzhu University, Jinan, China \\ ${ }^{2}$ School of Electrical and Computer Engineering and Birck Nanotechnology Center, Purdue University, West \\ Lafayette, Indiana, USA \\ Email: ${ }^{*}$ tpang@sdjzu.edu.cn
}

Received 24 March 2015; accepted 24 April 2015; published 27 April 2015

Copyright (C) 2015 by authors and Scientific Research Publishing Inc.

This work is licensed under the Creative Commons Attribution International License (CC BY).

http://creativecommons.org/licenses/by/4.0/

(c) (i) Open Access

\begin{abstract}
Nanoporous anodic aluminum oxide (AAO) with uniform and controllable pore diameters and periods over a wide range has been explored for various applications due to relatively easy fabrication processes. Moreover, one of the interesting possibilities afforded by the anodization process is that the anodization can take place on aluminum films with arbitrary shape, such as a section of cylinder or sphere, which has not yet been well studied or applied in nanofabrication. In this paper, we report that highly ordered conical nanohole arrays prepared by the anodization of cylindrical and spherical Al films have been fabricated. As can be seen by scanning electron microscopy (SEM), straight nanohole arrays have been grown along the radical directions of the cylindrical or spherical alumina membrane without bending or branching at all, the diameter of the conical nanoholes and the diameter change along individual channels can be tuned by changing the curvature of the membrane. These new types of templates may open new opportunities in optical, electronic and electrochemical applications.
\end{abstract}

\section{Keywords}

Nanoporous Anodic Aluminum Oxide, Conical Nanohole Arrays

\section{Introduction}

Anodic aluminum oxide (AAO) has generated considerable interest as a template for nanofabrication [1]-[3]. Porous AAO structures with flat surface are typically obtained by anodic oxidation of aluminum metal in solu-

*Corresponding author. 
tions of sulfuric, oxalic, or phosphoric acid [4] [5]. Flat AAO films have been extensively used as templates for the fabrication of nanowires or nanocables [6]-[11] and nanotubes [12]-[19], as well as masks for growth of other nanostructures such as anti-dots, nano-dots or nanopillars [20]-[23]. These nanomaterials exhibit unique optical, magnetic, or electronic properties and have potential applications in various areas, such as high-density magnetic recording media, optical devices, field emission displays, mechanical oscillators, energy storage, nanoelectrodes, and sensors. In addition to membranes with cylindrical channels, various other types of AAO nanostructures have also been fabricated and theoretically studied, such as triangular and square pore arrangements [24] [25], and taper hole arrays [26]. AAO film with micro zone of curved surface which were made by impurity induced or by methods of photolithography, focused ion beam (FIB) lithography has also been studied recently [27]-[29], Due to limitation of the methods they used, the area of the curved surface of AAO were only occurred in a small area while the most area of the surface including other side were still flat, and the nanoholes in the curved area were bending and branching. It still remains a great challenge to make the AAO membrane with large curved surface in both sides and the straight nanohole arrays without bending and branching, which are obviously demanded in many applications, such as solar cell [11].

In this paper, we report a simple method for fabricating cylindrical or spherical AAO membranes with highly ordered conical nanohole arrays, which was not possible previously. Straight nanoholes were grown along the radical directions of the cylindrical or spherical alumina without bending and branching at all. The diameter of the conical nanoholes and the diameter change along the individual nanohole can be tuned by changing the curvature of the membrane, either in one or two dimensions.

\section{Experimental}

Cylindrical or spherical AAO membranes with highly ordered straight conical nanohole arrays were prepared by two steps: (1) the generation of cylindrical and spherical Al films; (2) the generation of AAO by two-step anodization process, adapted Lyvers et al. [30]. Briefly, high-purity (99.999\%) flat aluminum films were placed on a cylinder or sphere to mould a given shape. Prior to anodization, the moulded films were electropolished for better pore ordering and uniformity. The films were then removed for anodization, which were carried out under a constant cell voltage of $40 \mathrm{~V}$ in a $0.3 \mathrm{M}$ oxalic acid solution at $10^{\circ} \mathrm{C}$ for $20 \mathrm{~h}$. The formed alumina was then removed by a $6 \mathrm{wt} \%$ phosphoric acid and $1.6 \mathrm{wt} \%$ chromic acid solution, and the cylindrical or spherical $\mathrm{Al}$ sheet was anodized again under the same conditions as described above, for $8 \mathrm{~h}$. After the second anodization, the remaining aluminum was removed in a saturated $\mathrm{HgCl}_{2}$ solution. A subsequent etching treatment was carried out in a $0.1 \mathrm{M}$ phosphoric acid solution at $30^{\circ} \mathrm{C}$ for $20 \mathrm{~min}$ to remove the aluminum oxide barrier layer on the bottom side of the AAO. Scanning electron microscopy was used to characterize the structure of the cylindrical or spherical AAO membranes.

\section{Results and Discussion}

Figure 1 show the large cylindrical AAO membranes before (a) and after (b) removing the remaining aluminum and the barrier layer. The radius of curvature is $0.35 \mathrm{~cm}$ (the radius of spherical AAO membranes is $1.25 \mathrm{~cm}$ in our experiments, not shown here).

For the cylindrical AAO, Figure 2(a) and Figure 2(b) show the SEM (SEM) JSM-6300) images of the nanohole arrays on the inner and outer surface, respectively. It can be clearly seen that highly ordered nanohole arrays were obtained in the surfaces of cylindrical AAO membrane. The statistical data from the different areas show that the diameter of nanohloe in the inner surface and the outer surface are $66.8 \mathrm{~nm}$ and $82.8 \mathrm{~nm}$, respectively. We can also see from the SEM images of the cross section (Figure 3) that the nanoholes are well-ordered without bending and branching at all. So, we conclude that the straightly conical nanohole arrays were obtained in the cylindrical AAO membranes. With the axis of the cylindrical surface as the symmetric line, conical hole arrays present a radial arrangement ordered along the normal vectors of the cylinder (Figure 4(a)), the radius change along the conical nanohole is $0.44 \mathrm{~nm} / \mu \mathrm{m}$ (the film is about $36 \mu \mathrm{m}$ thick). It is impossible to show the small change of diameter in one SEM image of the cross section.

SEM images of different AAO template with different cylinder radii show that there is a strong correlation between the maximum and minimum radius of the conical nanohole and the radius of curvature of the AAO template. This kind of correlation gives us an additional degree of freedom to control the diameter of nanoholes 


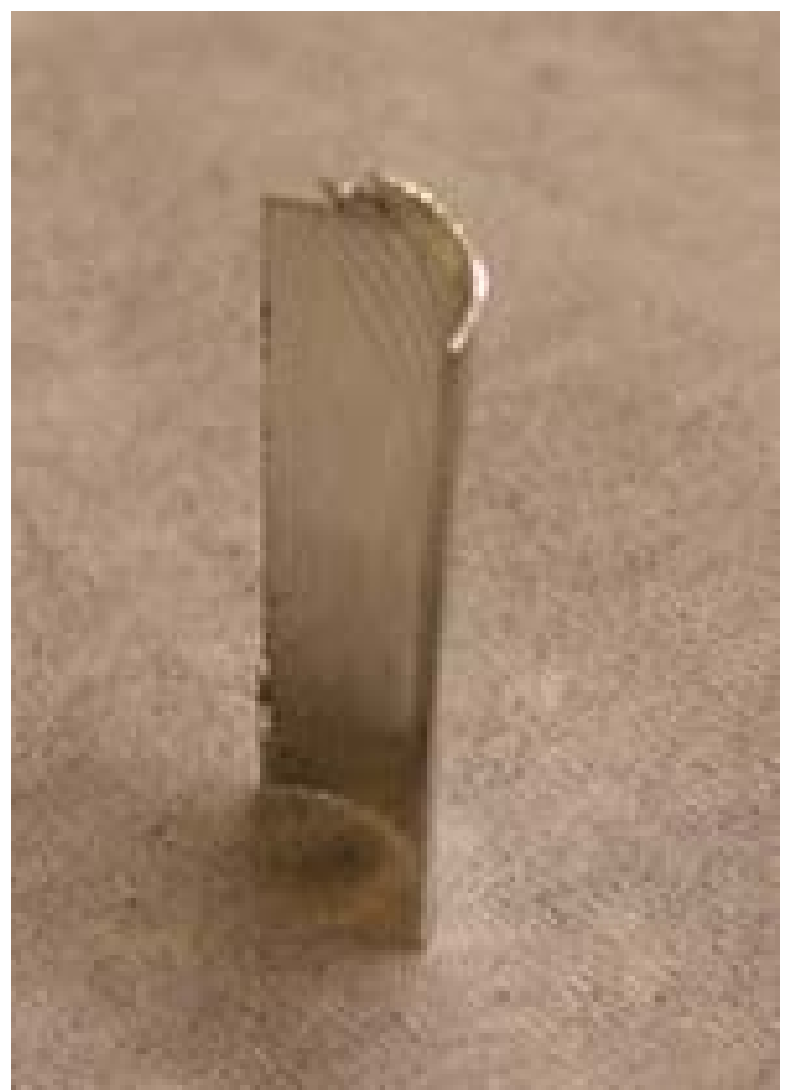

(a)

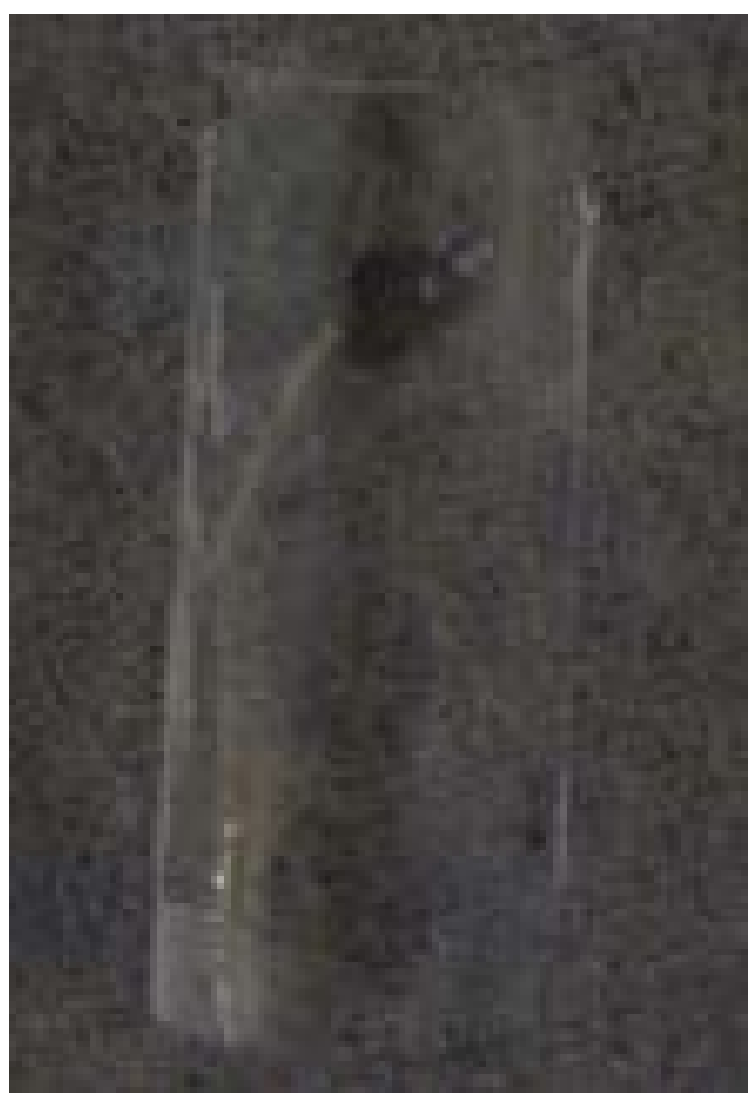

(b)

Figure 1. Large Cylindrical AAO membranes before (a) and after (b) removing the remaining aluminum and the barrier layer.

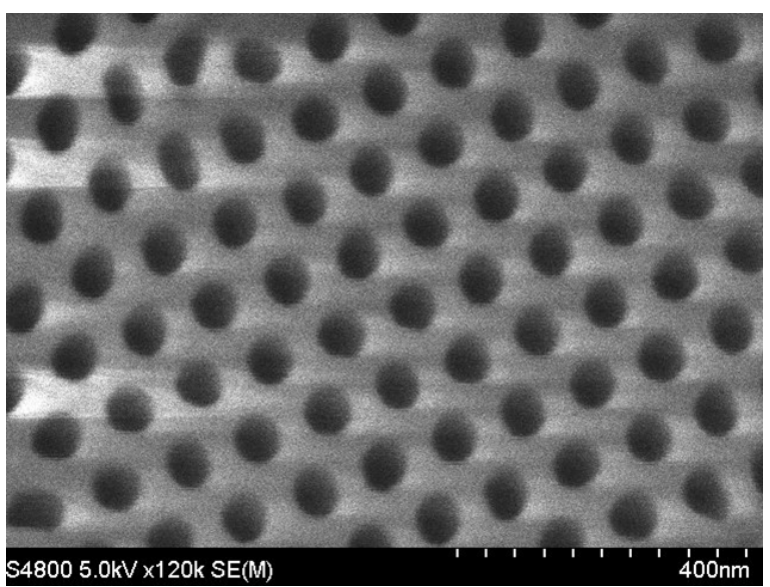

(a)

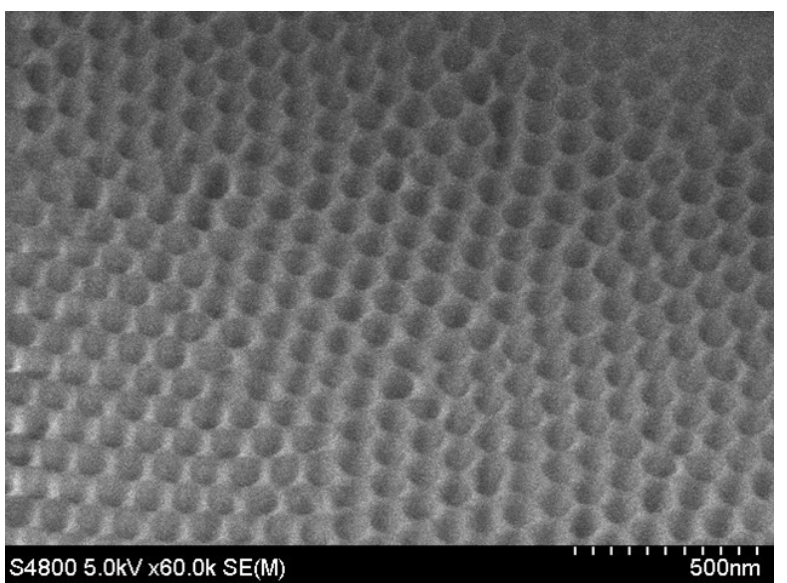

(b)

Figure 2. SEM images of the nanohole arrays of the cylindrical AAO membranes on the inner surface (a) and outer surface (b).

in curved AAO membranes than in flat AAO membranes, in which the change of the diameter of nanohole can only be realized by changing the anodization voltage or the concentration of the acid solution at the expense of the ordered arrangement of nanohole arrays.

However, there is also an indirect correlation ship between the radius change along the individual conical nanoholes and radius of cylinder; the radius change increases as the cylinder radius decreases. This level of control 


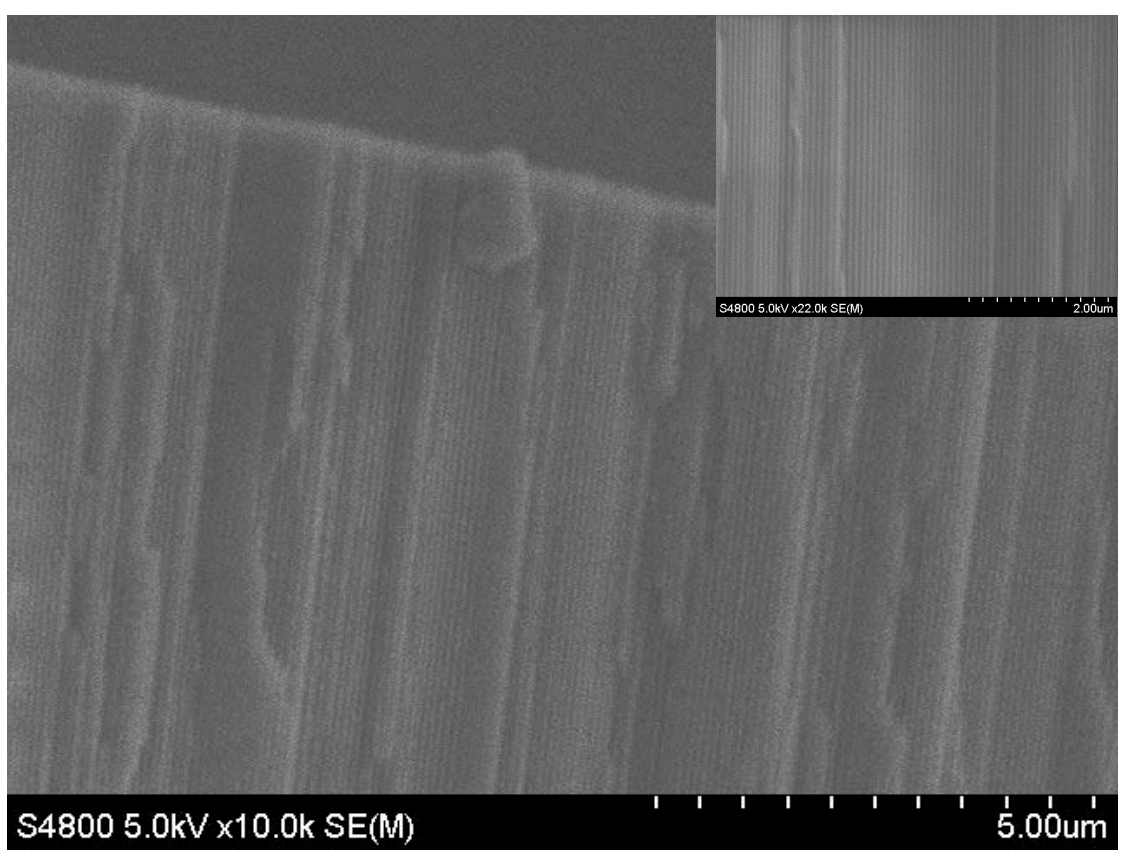

Figure 3. Cross section SEM image of the cylindrical AAO membranes showing the straight nanohole arrays with no branching and bending. The inset is the high magnification image of the cross section.

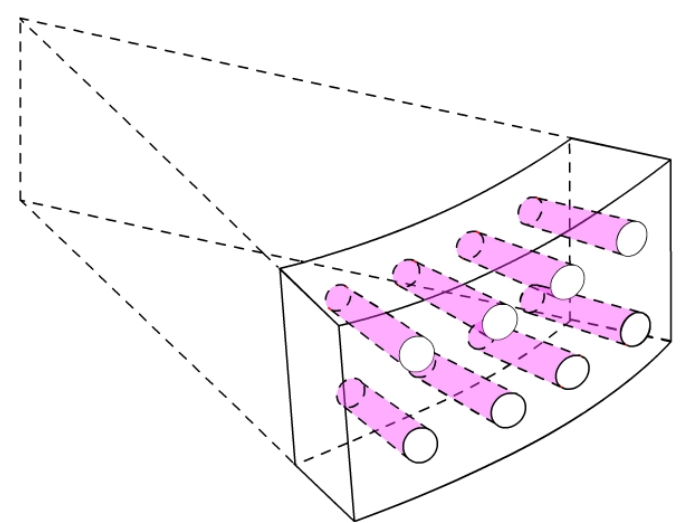

(a)

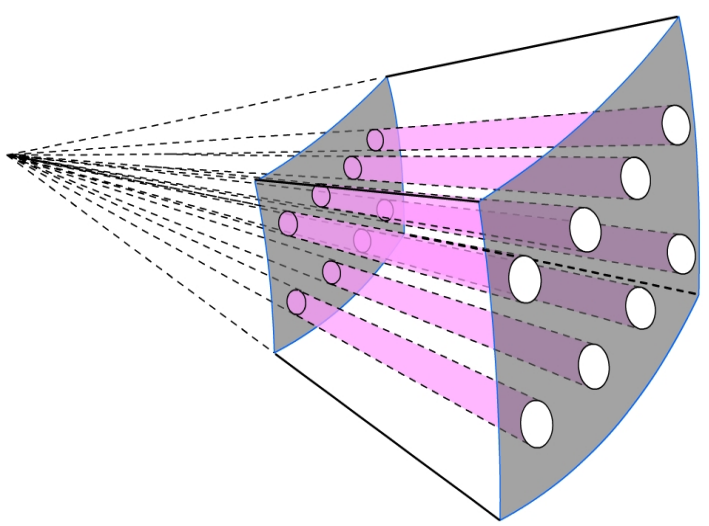

(b)

Figure 4. Schematic images of conical nanohole arrays in cylindrical (a) and spherical (b) AAO membranes.

of the nanoholes radius and conical structures enable us to tailor the properties of nanowire-based hyperbolic materials, used for sub-diffraction imaging or radiative decay engineering [31].

We get the same nanohole structures in the spherical AAO membrans as in the cylindrical AAO (Figure 4(b)). The only difference is that the symmetry center of the conical nanohole arrays is the center of the sphere instead of the axis of the cylinder.

It is worth to repeat again that in our results, the conical nanoholes are straight without bending and branching, which usually occurred in anodization of curved surface of $\mathrm{Al}$ [27]-[29]. As it is well known that pore formation is an electric-field-assisted process. The bending and branching phenomenon of the pore channel in curved $\mathrm{Al}$ surface were suggested due to unbalanced electric field, the different conductivities and the different interpore distance around the nanoholes [27]-[29]. In our experiments, however, it is important to note that the both sides of curved Al film were anodized simultaneously (i.e., we get two pieces of curved AAO membranes at a time), and there is neither $\mathrm{Al} / \mathrm{Si}$ interface nor the different conductivities between walls of neighbor nanoholes (In their experiments [27], however, the electric field will change in the immediate vicinity due to the different conductivities of $\mathrm{Al}$ and $\mathrm{Si}$ at the process of anodization). So, the uniform distribution of electric field on the both sur- 
faces of curved $\mathrm{Al}$ film remains in our experiments because of the symmetric anodization process, which is pretty much the same as the anodization of flat $\mathrm{Al}$ and this may be the main reason for the straight nanoholes without bending and branching.

Another notable features of the curved AAO membranes is that the radius of nanohole change along its growth direction, i.e., along the radial direction of the cylindrical or spherical membranes, which were not found before in flat AAO membranes. It is well known that all the pore channels initialize and propagate along the direction of electric field, i.e., normal to the Al surface [1]-[4] [27]-[29]. So, in our experiments, the nanohole with conical shape were formed because of the geometric symmetry and the symmetric anodization. That means we can control the diameter of nanohole by the thickness of curved AAO membranes (the anodization time, of course) and the curvature as mentioned above, and also the anodizaion voltage as well. It could be used as a new platform for nanofabrication.

Like typical flat AAO membranes, these curved AAO structures with radial arrangement of conical nanohole arrays can also be used as a template for carbon nanotube growth by chemical vapour deposition methods or for nanowire, nanocable and nanotube growth by sol-gel, electrodeposition or supercritical fluid approaches. By filling the nanopores with selected materials, these new structures have potential in optical, magnetic, and sensing applications. For example, the curved membranes with nanowires or nanotubes inside could be used for anti-reflection purposes with even optical response for much larger solid angles than flat ones. It has been predicted that a silver nanowire metamaterial in curvilinear AAO template with hyperbolic dispersion has a property of a hyperlens — the device allowing for sub-diffraction color imaging in a far field [26] [31].

\section{Conclusion}

In summary, a novel self-organized nanoporous AAO membranes with large cylindrical and spherical in shape, have been prepared and characterized carefully. Straightly conical hole arrays presents a radial arrangement ordered along the radial direction of the cylindrical or spherical membranes without bending and branching at all. More important, by tuning the radius of curvature of the AAO membranes and the anodization time, we can control the radius and the radius change rate of conical nanohole arrays. Our results are instructive for fundamental understanding of purposely designed curved AAO membranes; they will also greatly enhance the control of pore growth on non-planar faces surfaces. This will open opportunities in applications where the substrates need to be curve in shape, such as sensors, catalysts, and optic fibers.

\section{References}

[1] Masuda, H., Yada, K. and Osaka, A. (1998) Self-Ordering of Cell Configuration of Anodic Porou Alumina with Large-Size Pores in Phosphoric Acid Solution. Japanese Journal of Applied Physics, 37, L1340-L1342.

[2] Martin, C.R. (1994) Nanomaterials: A Membrane-Based Synthetic Approach. Science, 266, 1961-1966. http://dx.doi.org/10.1126/science.266.5193.1961

[3] Thompson, G.E. and Wood, G.C. (1981) Porous Anodic Film Formation on Aluminium. Nature, 290, $230-232$. http://dx.doi.org/10.1038/290230a0

[4] Masuda, H. and Fukuda, K. (1995) Ordered Metal Nanohole Arrays Made by a Two-Step Replication of Honeycomb Structures of Anodic Alumina. Science, 268, 1466-1468. http://dx.doi.org/10.1126/science.268.5216.1466

[5] Cheng, C. and Ngan, A.H.W. (2013) Fast Fabrication of Self-Ordered Anodic Porous Alumina on Oriented Aluminum Grains by High Acid Concentration and High Temperature Anodization. Nanotechnology, 24, 215602-215612. http://dx.doi.org/10.1088/0957-4484/24/21/215602

[6] Lakshmi, B.B., Dorhout, P.K. and Martin, C.R. (1997) Sol-Gel Template Synthesis of Semiconductor Oxide Microand Nanostructures. Chemistry of Materials, 9, 2544-2550. http://dx.doi.org/10.1021/cm970268y

[7] Yin, A.J., Li, J., Jian, W., Bennett, A.J. and Xu, J.M. (2001) Fabrication of Highly Ordered Metallic Nanowire Arrays by Electrodeposition. Applied Physics Letters, 79, 1039-1041. http://dx.doi.org/10.1063/1.1389765

[8] Nicewarner-Pena, S.R., Freeman, R.G., Reiss, B.D., He, L., Pena, D.J., Walton, I.D., Cromer, R., Keating, C.D. and Natan, M.J. (2001) Submicrometer Metallic Barcodes. Science, 294, 137-141. http://dx.doi.org/10.1126/science.294.5540.137

[9] Pang, Y.-T., Meng, G.-W., Zhang, L.-D., Qin, Y., Gao, X.-Y., Zhao, A.-W. and Fang, Q. (2002) Arrays of Ordered Pb Nanowire and Its Optical Properties for Laminated Polarizers. Advanced Functional Materials, 12, 719-722. http://dx.doi.org/10.1002/1616-3028(20021016)12:10<719::AID-ADFM719>3.0.CO;2-J 
[10] Fan, Z.Y., Razavi, H., Do, J.-W., Moriwaki, A., Ergen, O., Chueh, Y.-L., Leu, P.W., Ho, J.C., Takahashi, T., Reichertz, L.A., et al. (2009) Three-Dimensional Nanopillar-Array Photovoltaics on Low-Cost and flexible Substrates. Nature Materials, 8, 648-653. http://dx.doi.org/10.1038/nmat2493

[11] Fan, Z.Y., Kapadia, R., Leu, P.W., Zhang, X.B., Chueh, Y.L., Takei, K., Yu, K., Jamshidi, A., Rathore, A.A., Ruebusch, D.J., et al. (2010) Ordered Arrays of Dual-Diameter Nanopillars for Maximized Optical Absorption. Nano Letters, 10, 3823-3827. http://dx.doi.org/10.1021/nl1010788

[12] Che, G., Lakshmi, B.B., Fisher, E.R. and Martin, C.R. (1998) Carbon Nanotubule Membranes for Electrochemical Energy Storage and Production. Nature, 393, 346-349. http://dx.doi.org/10.1038/30694

[13] Li, J., Papadopoulos, C., Xu, J.M. and Moskovits, M. (1999) Highly-Ordered Carbon Nanotube Arrays for Electronics Applications. Applied Physics Letters, 75, 367-369. http://dx.doi.org/10.1063/1.124377

[14] Li, J., Papadopoulos, C. and Xu, J.M. (1999) Y-Junction Carbon Nanotubes and Controlled Growth. Nature, 402, 253-254.

[15] Suh, J.S., Lee, J.S. and Kim, H. (2001) Linearly Joined Carbon Nanotubes. Synthetic Metals, 123, 381-383. http://dx.doi.org/10.1016/S0379-6779(01)00316-2

[16] Jeong, S., Hwang, H., Lee, K. and Jeong, Y. (2001) Template-Based Carbon Nanotubes and Their Application to a Field Emitter. Applied Physics Letters, 78, 2052-2054. http://dx.doi.org/10.1063/1.1359483

[17] Hu, W., Yuan, L., Chen, Z., Gong, D. and Saito, K. (2002) Fabrication of Nanoelectrodes Using Porous Alumina Nanotemplates. Journal of Nanoscience and Nanotechnology, 2, 203-207.

[18] Steinhart, M., Wehrspohn, R.B., Gösele, U. and Wendorff, J.H. (2003) Nanotubes by Template Wetting: A Modular Assembly System. Angewandte Chemie International Edition, 43, 1334-1344. http://dx.doi.org/10.1002/anie.200300614

[19] Choi, W.B., Bae, E., Kang, D., Chae, S., Cheong, B.H., Ko, J.H., Lee, E. and Park, W. (2004) Aligned Carbon Nanotubes for Nanoelectronics. Nanotechnology, 15, S512. http://dx.doi.org/10.1088/0957-4484/15/10/003

[20] Crouse, D., Lo, Y., Miller, A.E. and Crouse, M. (2000) Self-Ordered Pore Structure of Anodized Aluminum on Silicon and Pattern Transfer. Applied Physics Letters, 76, 49-51. http://dx.doi.org/10.1063/1.125652

[21] Liang, L., Chik, H., Yin, A. and Xu, J. (2002) Two-Dimensional Lateral Superlattices of Nanostructures: Nonlithographic Formation by Anodic Membrane Template. Journal of Applied Physics, 91, 2544-2546. http://dx.doi.org/10.1063/1.1433173

[22] Nishioa, K. and Masuda, H. (2004) Dependence of Optical Properties of Ordered Metal Hole Array on Refractive Index of Surrounding Medium. Electrochemical and Solid-State Letters, 7, H27-H28. http://dx.doi.org/10.1149/1.1759294

[23] Goh, C., Coakley, K.M. and McGehee, M.D. (2005) Nanostructuring Titania by Embossing with Polymer Molds Made from Anodic Alumina Templates. Nano Letters, 5, 1545-1549. http://dx.doi.org/10.1021/nl050704c

[24] Masuda, H., Asoh, H., Watanabe, M., Nishio, K., Nakao, M. and Tamamura, T. (2001) Square and Triangular Nanohole Array Architectures in Anodic Alumina. Advanced Materials, 13, 189-192. http://dx.doi.org/10.1002/1521-4095(200102)13:3<189::AID-ADMA189>3.0.CO;2-Z

[25] Ergen, O., Ruebusch, D.J., Fang, H., Rathore, A.A., Kapadia, R., Fan, Z.Y., Takei, K., Jamshidi, A., Wu, M. and Javey, A. (2010) Shape-Controlled Synthesis of Single-Crystalline Nanopillar Arrays by Template-Assisted Vapor-LiquidSolid Process. Journal of the American Chemical Society, 132, 13972-13974. http://dx.doi.org/10.1021/ja1052413

[26] Kawata, S., Ono, A. and Verma, P. (2008) Subwavelength Colour Imaging with a Metallic Nanolens. Nature Photonics, 2, 438-442. http://dx.doi.org/10.1038/nphoton.2008.103

[27] Yin, A.J., Guico, R.S. and Xu, J. (2007) Fabrication of Anodic Aluminium Oxide Templates on Curved Surfaces. Nanotechnology, 18, Article ID: 035304. http://dx.doi.org/10.1088/0957-4484/18/3/035304

[28] Chen, B. and Lu, K. (2011) Influence of Patterned Concave Depth and Surface Curvature on Anodization of Titania Nanotubes and Alumina Nanopores. Langmuir, 27, 12179-12185. http://dx.doi.org/10.1021/la202559h

[29] Chung, C.K., Liao, M.W., Lee, C.T. and Chang, H.C. (2011) Anodization of Nanoporous Alumina on Impurity-Induced Hemisphere Curved Surface of Aluminum at Room Temperature. Nanoscale Research Letters, 6, 596. http://dx.doi.org/10.1186/1556-276X-6-596

[30] Lyvers, D.P., Moon, J.M., Kildishev, A.V., Shalaev, V.M. and Wei, A. (2008) Gold Nanorod Arrays as Plasmonic Cavity Resonators. ACS Nano, 2, 2569-2576. http://dx.doi.org/10.1021/nn8006477

[31] Barnakov, Yu.A., Kiriy, N., Black, P., Li, H., Yakim, A.V., Gu, L., Mayy, M., Narimanov, E.E. and Noginov, M.A. (2011) Toward Curvilinear Metamaterials Based on Silver-Filled Alumina Templates. Optical Materials Express, 1, 1601-1604. http://dx.doi.org/10.1364/OME.1.001061 